\title{
Why is Research Needed on Simulation to Enhance Plastic Surgery Education and Training?
}

\author{
Corné P.G. Nel \\ Private practice, Gauteng; \\ Health Professions \\ Education Programme, \\ Faculty of Health Sciences, \\ University of the Free State, \\ Bloemfontein, South Africa
}

\author{
Mathys J. Labuschagne \\ Clinical Simulation and \\ Skills Unit, School of \\ Medicine, Faculty of Health \\ Sciences, University of the \\ Free State, Bloemfontein, \\ South Africa
}

\author{
Gert J. van Zyl \\ Faculty of Health Sciences, \\ University of the Free State, \\ Bloemfontein, South Africa
}

\begin{abstract}
Changes in health care triggered major shifts in health sciences education, including a move to simulation in education and training. Simulation enhances student learning, provides controlled and safe practice opportunities, and shapes the acquisition of doctors' clinical skills/professional competence. Myriad research opportunities exist in the field of simulation-based medical education (SBME). The overall goal of this research was to reflect on the purpose and value of research on simulation and was aimed at improving plastic surgery education and training (teaching, learning and professional competence). The methodology is based on the conceptualisation and contextualisation of SBME. This article offers recommendations on the role and value of simulation in education; the enhancement of learning; integrating simulationbased education into training curricula; standardising plastic surgery training globally; the revalidation of competency in continuing medical education, and the engagement of teachers and students in educational research. Simulation has potential to play an integral role in developing better and safer health care services for patients worldwide.
\end{abstract}

\section{Introduction}

The role of simulation in surgical training is now beyond 'proof of concept' stages. The advantages of simulation in modern healthcare education and training systems have been well described in reports establishing the validity and transferability of skills learned in simulated clinical setting environments with demonstrable advantages to the system and patients [1]. Changes in health care triggered major shifts in health sciences education, including a worldwide move to utilising simulators in education and training [2]. According to Issenberg et al. [3], "...for instance, in the United States, the pressures of managed care are shaping the form and frequency of hospitalisations, resulting in higher percentages of acutely ill patients and shorter in-patient stays". A consequence of this is that medical students may have to forfeit opportunities to assess patients suffering from diverse and sometimes rare diseases and presenting with diverse symptoms. Efforts to increase cost-efficiency can no longer counter the decrease in training staff remuneration and financial resources available for education and training, resulting in less time spent on training at all levels and doctors having to find other ways to stay at the forefront of skills in and new knowledge on cases with which they are confronted.

Evidence of the value of simulation in education resulted in increased reliance on simulation technology to facilitate teaching and training innovation and enhancement of student learning and the mastering of skills, to provide controlled and safe practice opportunities, and to shape the acquisition of doctors' clinical skills/professional competence [2]. Simulation empowers students to make decisions regarding diagnostic and therapeutic procedures, and to experience the full impact of success and mistakes in a safe and authentic educational environment [2],[3].

Simulation-based medical education is an education and training strategy that makes use of simulation to bridge the gap between theory and practice in medical education. In terms of medical simulation, the word simulation means the "imitation of the operation of a real-world process or system over time" [4].

In medicine this may mean any process and/or system designed and planned to recreate an authentic clinical context and environment, providing 
opportunities for a student to assume a role of responsibility. The intention is to facilitate meaningful clinical experiences in a safe environment that the students can refer to and transfer to authentic clinical contexts [5] that is to educate and train students in a non-threatening, simulated environment, providing opportunities for experimentation and practising in a non-threatening environment. Here students can be brought to the level of application, taking them into the realm of real-word circumstance and helping them to overcome the gap between theory and reality, and to experience a feeling of what it is to be working with real patients in a real-world context. This plays an important role in reflection and the integration of theory with skills. Simulation offers a safe environment and context where students hone their skills and determine for themselves what their strengths and weaknesses are and what they should do about the areas they need to improve in and build out, and they build self-confidence.

Soltanian [6] defines simulation "as the replication of a real-world process or system over time" and makes an appeal to surgeon educators to employ their knowledge and skills in developing and enhancing simulators for training the next generation of plastic surgeons. The required technology has become more readily available lately and surgical simulations have improved significantly in recent years [6]. The most common types of models in surgical simulation include physical models (e.g. to train fundamentals of laparoscopic surgery); mathematic models (e.g. commonly used within a computer simulation); graphical models (e.g. graphical depiction of an object or system), and finite element models (e.g. large and complex systems are divided into smaller and simpler parts with more predictable behaviour with a view to replicating the more complex objects [6].

A simulator is a model that encapsulates the key features or behaviours of an identified process or system found in the real world [4]. A medical simulator, therefore, demonstrates a key clinical characteristic or set of clinical responses or situations that mimic real-life conditions and responses. Medical simulators include computer programmes, part-task trainers, human patient simulators (or full-scale mannequins), and standardised patients [7].

An increasing number of students enter medical schools each year, with the consequence of more students competing for clinical cases and the number of conditions health care professionals are expected to attend to (case mix), as well as the shorter duration of hospitalisation of patients deprive students of opportunities to do clinical work. Simulation is excellently suited to cover this hiatus in medical training. Patients nowadays are better informed, have greater expectations and may exercise their right not to be involved in student education, resulting in an even smaller teaching platform.

Issenberg et al. [3] identify five factors contributing to the increase in the use of simulations in medical education, namely a shortage of clinical education opportunities for clinical teaching; innovative technology for diagnosis and management; assessment of professional competence; medical errors; patient safety and team training; and the role of intentional practising. Due to the pressure caused by these factors, the burden of proof for adoption need not rely on randomized control trials, but rather, opportunities exist for a wide range of studies making use of simulation. Thus, investigators using these opportunities can contribute new knowledge in the field of simulation-based medical education, and, more specifically, its use in plastic surgery education and training.

Scalese [8] highlights the trend to utilise simulators for teaching, learning and assessment. It is posited that simulation-based medical education (SBME) plays a significant role in minimising risk to patients and enhancing medical training [9]. These authors [9] also posit that medico-legal issues and demands for accountability may be critical driving forces for the incorporation of simulation training in health care education.

\section{Problem statement and aim of the study}

Training in plastic surgery is not exempted from the drivers for the use of simulation mentioned. The increased competition for surgical exposure and practice, combined with smaller teaching platforms and shorter training times might have an impact on the quality and surgical competence of the registrar leaving the training programme. Plastic surgery is falling behind other disciplines in adopting simulation-based medical education, as in many areas and disciplines great strides are made in implementing simulation in formal training programmes, with leaders in the field anaesthesia, emergency medicine and laparoscopic surgery.

A number of articles recently have been published on topics such as the use and potential use, as well as the importance of simulation in plastic surgery [10], [11]; the integration of surgical simulation in plastic surgery residency training [12]; and skills transferred to the operating room by surgical simulation [13]. In response two seemingly contradictory goals in education have been put forward as priorities. On the one hand, there is a push for further standardisation of education. To this end, the Accreditation Council for Graduate Medical Education (ACGME) and the American Board of Medical Specialities (ABMS) 
have defined six core competencies required of all residents [10]. The standardization of aims will increase patient safety by reducing surgical errors and improving the quality of care, while at the same time maximizing hospital resources. On the other hand, the medical education model ought to allow for individualisation to reflect the fact that people obtain knowledge and master competencies differently. According to this line of reasoning there should be room for one student's path to differ from another's to best accommodate the students' learning styles [11].

Satava [14] cites three concepts that will be key in revolutionising medical education that exemplifies these dual priorities: increased efficiency in education by standardising curricula; individualisation of education, and moving from time-based training to competency-based training.

Substantial opportunities exist for researchers to contribute to new knowledge in the field of simulation-based medical education (SBME) and, more specifically, plastic surgery education. Medicolegal issues and demands for accountability are critical driving forces for the incorporation of simulation training in health care education.

To generate research evidence in education and to establish a research foundation it is necessary to consider/reflect on the purpose of research directives in the specific field of education. Cook, Bordage and Schmidt [15] explored the research performed in Health Professions Education and suggest a framework for classifying the purposes of educational research. According to Cook et al. [15], indications are that research in medical education does not necessarily inform educational practice. These authors [15] mention that a best-evidence medical education (BEME) review by Issenberg et al. [3] found weak evidence supporting several conditions for effective high-fidelity simulation, but noted that few or no strong studies could be found. However, in the BEME guide Features and uses of high-fidelity medical simulations that lead to effective learning, Issenberg et al. [16] clearly state that research in this field "needs improvement in terms of rigor and quality. High-fidelity medical simulations are educationally effective and simulation-based education complements medical education in patient care settings". As far as research directives are concerned, Issenberg et al. [16] are of the opinion that the "lack of unequivocal evidence for much of the research on simulation-based medical education clearly calls for better research and scholarship in medical education".

Cook et al. [15] classify the purposes of research in a useful framework to understand and to give meaning to the research process, as well as to identify potential solutions consisting of three categories, namely description, justification and clarification. Description studies focus on the first step in the scientific method, namely observation, and asks: "What was done?"; Justification studies focus on the final step in the scientific method comparing educational interventions with each other to address the question: "Did it work?". Clarification studies employ each step in the scientific method, starting with observations (building on prior research) and models or theories, making predictions, and testing these predictions. Such studies ask the questions: "How does it work?" and "Why does it work?" [15].

A scientific approach and clear, direction-giving research that will advance the science in the specific field of study, namely plastic surgery and simulation, therefore, are not negotiable. It thus is imperative that any research directive suggested here has to follow this deeper approach when executed during different research initiatives and projects.

Nel, Labuschagne and van Zyl [17] at the IICE conference in 2016 in Ireland emphasised the importance of a scientific proposal with a clear agenda expressing the need for research in the field of plastic surgery and simulation, proposing research topics in a systematic way.

The problem addressed in the research reported here thus was a lack of profound research about the use of simulation in the education and training of plastic surgeons. The study was aimed at identifying aspects to be included as a research directive for studies conducted with a view to improving teaching, learning and professional competence in plastic surgery education and training. The methodology was based on the conceptualisation and contextualisation of SBME.

\section{Residency programmes and simulation}

Worldwide, different models exist for education and training in plastic surgery, including the models of learning through an apprenticeship relationship with senior clinical colleagues, own observation, or self-directed learning - motivated by a candidate's own internal drive. In some cases, registrars receive little guidance in terms of the knowledge, competencies, skills and attitudes they are expected to acquire during residency. Residency programmes are responsible for producing technically competent surgeons, but not all of the necessary procedural skills are truly mastered during these training periods. "Classroom training" does not translate into effective procedural skills and competence does not always match confidence. Although residency programme directors are required to attest to the competency of recent graduates, they cannot possibly evaluate the performance of every procedure carried out by every 
resident. A further shortcoming is that systematic evaluation using structured objective criteria seldom is used to establish procedural competence, and except for a few procedures, it cannot be determined and prescribed how many times a specific person should repeat a specific procedure to ensure competence.

Rosen, Long, McGrath and Greer [18] point out that in contrast to the traditional apprenticeship model, twenty-first century surgical training is characterised by an increasingly objective, standardised approach using equipment such as simulators to optimise patient safety, surgical care, and hospital resources, and minimise errors. The driving forces behind these changes are developments in medical error statistics, evidence-based medicine, and fewer attending hours. Through increased accuracy simulation can improve results and also lower risk and procedure cost because of fewer procedures and less operating room time [18].

The Accreditation Council for Graduate Medical Education (ACGME) and the American Board of Medical Specialties (ABMS) identify six core competencies for residents, namely

"Patient care, medical knowledge, practice-based learning and improvement, inter-personal and communication skills, professionalism, and systems-based practice” [10].

Training thus has evolved from traditional apprenticeship to more objective, standardised approaches. A shift towards competency-based surgical training comes with two key concepts: objective assessments and simulation laboratory training. The time thus has come for residency programmes to explore and expand their use of simulation.

A joint initiative of the ACGME and the ABMS, The Plastic Surgery Milestone Project, compiled descriptors and targets for resident performance, based on the above-mentioned core competencies which may be categorised at five training levels, moving from Level 1 where the resident demonstrates the mastering of milestones that new residents have to achieve to Level 5 where the resident has advanced beyond performance targets the residents have to meet during residency and before graduation [19].

The American College of Surgeons (ACS) has decided to introduce simulation in training and education for general surgery in three phases: Skills training, procedure training, and team training. Mittal et al. [10] recommend that plastic surgery should follow this simulation initiative with modifications appropriate to the specialty. Phase 1, Skills, is attended to in the resident's general surgery training, but Phase 2, Procedures, focuses on the development of procedures specific to plastic surgery. For Phase 3,
Competencies in teamwork, the competencies for plastic surgery resemble those for general surgery and include team-training simulators to improve communication in emergency departments, clinics, operating rooms, and hospital wards.

Arbogast and Rosen [11] in their article, Simulation in Plastic Surgery Training: Past, Present and Future, propose that this three-phase strategy be adapted for plastic surgery residency by modifying it to address challenges specific to the field. They are of the view that a unified commitment by medical educators is required to use simulation " $[\mathrm{t}] \mathrm{o}$ simultaneously standardize the training curriculum, individualize the method of acquiring information, and objectively evaluate the training process".

\section{Aspects of simulation}

Simulation is a useful aid for honing skills and bridging the gap between theory and practice in a variety of teaching, training, learning, and assessment situations.

\subsection{In which ways can simulation be of help?}

Simulation can play a valuable role in improving patient safety, facilitating better surgeon-patient interaction, maximizing hospital resources, lowering risks through refining precision, improving results by demanding fewer procedures and decreasing operating room time and procedure cost.

Simulation shows great promise to change teaching methods - the traditional model of see one, do one, teach one is an inefficient and risky approach to acquiring technical skills and competencies. This, coupled with public demand for patient safety and an increasing reluctance to be "practised" on, has created ideal opportunities for simulation-based medical education to improve teaching.

Simulation methodologies enable tailored training interventions in a low-threat environment. Limited exposure to patients with low-incidence and highcomplexity conditions may be addressed by means of simulation and render competency as outcome. Simulation also may be used to assess the outcome of training in a more objective and structured way. This concept is used in the development of the objective structured clinical examination (OSCE). An adapted version of the OSCE was created to assess technical skills. This is called the objective structured assessment of technical skills (OSATS).

Neumeister [20] mentions that many plastic surgery programmes find it problematic to balance clinical, hands-on education and training with their didactic programme. Work and duty hour restrictions add another layer of complexity to surgical education. Furthermore, the ultimate step in learning is not the 
transmission of information from the teacher to the learner but rather the processing of the information by the learner [20]. According to Neumeister [20] the current trends in educational technology "include internet-based instruction, hands-on simulation devices, mobile devices, virtual and augmented reality, point-of-care learning and assessment, and learning analytics". Telemedicine combined with holograms brings distant expertise into the classroom for learners at all levels. Before entering the operating room, interactive mobile applications allow the residents opportunities to practise procedures over and over again to solidify the understanding of each aspect of any given surgery skill [20]. "Educators need to use innovation and technology to make the best use of data and knowledge to train next generations of plastic surgeons. Educational technology offers unique tools to help learners acquire and process the information needed to become masters of their surgical specialty" [20].

\subsection{Formative versus summative OSATS}

Both formative and summative OSATS provide an excellent opportunity for feedback and reflection on observed performance. Some argue that the best predictor of the quality of performance is repetitive or deliberative practice - with supervisors providing corrective feedback until the skill is mastered. Formative OSATS thus represent an area in which simulation excels.

\subsection{In which areas can simulation be implemented?}

Simulation-based medical education can target different levels for intervention. It has a role to play at individual level (e.g. supplementing clinical experience, procedural simulation and task training), team and unit level (e.g. behavioural training, multidisciplinary team interactions, and debriefing), as well as at an organisational level (e.g. on-site simulation to identify vulnerabilities in specific processes as well as broader systems, and disaster management).

\subsection{Studying and improving performance}

A critical on-going issue is identification and remediation of individuals who are underperforming. The simulation laboratory setting may help determine if an individual's deficits lie in history and examination taking, other data-gathering skills, synthesis, decision making or prioritization. In this setting the following may also be determined: Physical ability, lack of practice, effect of fatigue, or other similar areas that may contribute to underperformance. This is an often-underutilised application of simulation providing ample opportunity for future research.

\subsection{Priorities for simulation-based medical education in plastic surgery and recommendations}

Based on the foregoing the following, as an example, may be regarded as priorities for simulationbased medical education in plastic surgery. After each set of priorities a recommendation is provided for the realisation of the priorities:

\section{Integration into training curricula}

- The formal integration of simulation into curricula: It does not suffice to use simulation on a voluntary basis without protected simulation time. Very few students make use of simulation facilities if simulation is not formally integrated in the programme.

- Simulation should be synchronised with clinical training - there is little benefit if simulation time clashes with clinical teaching times.

- Ideally, simulation should be implemented at multiple tiers, namely skills rotations (must be completed to progress to next block/year), independent study/practice opportunities to allow students to progress/become proficient at their own pace, as well as evaluation and assessment of competency.

In order for the above to be achieved, the research question that must be answered is: Can simulation in postgraduate plastic surgery education and training enhance the effectiveness of learning in this discipline?

Standardisation of plastic surgery training globally

- SBME should be employed to standardise plastic surgery training, due to the large variation in the scope of plastic surgeons worldwide.

- SBME should be employed to counter a lack of clinical exposure opportunities, or (especially in the Third-World setting) programmes overburdened by a specific workload (for example, burns and burn reconstruction), which limits the time and resources available for exposure to other areas of the discipline (for example aesthetic surgery).

- SBME should set a basic standard that might enable educators and researchers to compare and contrast different training programmes worldwide.

- SBME should be available at all training facilities for plastic surgeons to enable the discipline to determine a list of basic core skills and competencies which every plastic surgeon should master. 
To achieve this, research is required to determine whether simulation might be useful in addressing the problem of a lack of opportunities for clinical exposure and practice.

\section{Skill maintenance and validation}

- Simulation should play an important role in revalidation of competency on a continuing medical education basis. Currently, in most CME programmes, the focus is on theoretical knowledge, but there is a large gap between theory and validating and revalidating surgical competency. Simulation could fill this gap.

- Safe thresholds of surgical skill should be identified, validated and may then be used and implemented using simulation as the vehicle to help achieve this goal.

- On-going skills maintenance programmes should be developed in which voluntary hours spent on simulators could be used to assist in skill maintenance, and with sufficient logging, could perhaps obviate the need to go for formal revalidation. To realize these priorities, the use of simulation stands in want of further research to facilitate the transfer of skills to real-world practice to assess the validity and reliability of procedural, clinical, and behavioural competency evaluation methods, and to determine the applicability of simulation to achieve these.

\section{Challenges in simulation-based medical education and research}

Simulation, like other methods in medical education, has some limits and pitfalls that should be kept in mind. These challenges provide ample opportunity for research, and we strongly advocate for research to be undertaken in these areas. The first challenge would be to utilise the wealth of research opportunities in plastic surgery on procedural competence and to establish the benefit, if any, of deliberate practice in a simulation-based setting. The discipline also lends itself very well to research on defining minimum levels of competency, and the role of simulation in maintaining and validating these levels. The problem of availability of facilities for simulation poses a challenge in itself, as well as the general misconception that simulation needs to be hifidelity, hi-tech, and expensive to be worthwhile. In the light of the resource demands of some simulation approaches, we should be susceptible to all solutions that comply with educational requirements. Furthermore, simulation cannot replace vital clinical actual observation and practical acquaintance with and learning from actual surgical practice. This, combined with the possible perception from previously trained professionals, who might feel intimidated and vulnerable ('Are they saying that our training was inferior?') forms a formidable stumbling block, which will have to be addressed in order for simulation-based medical education to be incorporated in training curricula. The challenge to succeed in this regard is found in meaningful involvement with trainers and trainees alike before embarking on simulation-based education.

\section{Future directions}

Work needs to be done on developing case libraries of simulation-based scenarios which have been peer reviewed. This will facilitate the promotion of simulation-based training by encouraging multicentre efforts and providing the opportunity for educational mentoring. Multi-centre simulation research efforts will be required to further develop and share evaluation tools specifically developed for resident and fellowship evaluations, where research on the transfer of skills into the real world takes precedence, as well as studies of valid and reliable assessment of procedural, clinical and behavioural competency evaluation.

\section{Discussion}

The research on simulation needs to include aspects such as the role and value of simulation in education; the enhancement of student learning at different cognitive levels; the integration of simulation-based education in teaching and training curricula; the standardisation of plastic surgery training globally; the revalidation of competency in continuing medical education, and the engagement of teachers and students in educational research. Medical simulation promises revolutionizing health care education, and specifically education in plastic surgery - but more work is required. Despite the development of various models and simulation-based learning tools used in plastic surgery training, the role of simulation in the specialty's training curriculum is not yet fully established. It is essential for simulationbased training to be an integral part of formal plastic surgery training programmes and be funded as such. It is also crucial to develop a skilled cadre of trainers and educators in well-managed simulation facilities. Medical simulation techniques have shown great promise in other specialities - we have outlined here the challenges and opportunities of realizing this promise in plastic surgery. 


\section{Conclusion}

The purposes for developing research directives are to compile theoretical and conceptual frameworks for the conception and design of research studies. The ensuing better understanding of the teaching and learning process will positively influence practice, clinical expertise and professional competence.

Further research is required to enhance the role of simulation in plastic surgery training to the benefit of performance evaluation, standardisation, certification and revalidation. Simulation as integral part of plastic surgery training is the preferred way to go to foster a move towards improved and safer plastic surgery services to the benefit of patients. Simulation in health care education provides ample opportunity for research. Medical simulation promises to revolutionise health care education provided that a skilled cohort of educators be developed in wellcoordinated simulation facilities. Simulation has the potential to play an integral role in developing better and safer health care services for patients worldwide, avoiding risk and providing real-life opportunities for students to hone their skills and enhance selfconfidence. It is clear that a scientific proposal to express the need for research in the field of plastic surgery and simulation, with clear directives proposing research topics in a systematic way urgently needs to be developed and made available to researchers investigating ways in which to ensure validity, reliability and viability in the education, training and assessment of plastic surgeon students. . It is hoped that aspects discussed in this article will contribute to and lay the foundation for such a research framework.

\section{References}

[1] C.R. Larsen, J Oestergaard, B.S. Pttesen and J.L. Soerensen. "The efficacy of virtual reality simulation training in laparoscopy: A systematic review of randomized trials", Acta Obstetricia et Gynecologica Scandinavica, 2012, 91 (9), pp. 1015-1028.

[2] S.B. Issenberg, W.C. McGaghie,, I.R. Hart, J.W. Mayer, J.M. Felner, E.R. Petrusa, R.A. Waugh, D.D. Brown, R.R. Safford, I.H. Gessner, D.I. Gordon, and G.A. Ewy, "Simulation technology for health care professional skills training and assessment", JAMA, 282(9), 1999, pp. 861-866. http://www.ncbi.nlm. nih.gov/pubmed/10478693. (Accessed date: 2 April 2015).

[3] S.B. Issenberg, W.C. McGaghie, E.R. Petrusa, D. Lee Gordon, and R.J. Scalese, "Features and uses of high-fidelity medical simulations that lead to effective learning: A BEME systematic review", Med Teach 27(1), 2005, pp. 10-28.

[4] Banks, J. and Georgia, M. Discreet event simulation, 1999. http://www.telecom.otago.ac.nz/ tele302/ref/banks DES.pdf. (Accessed date: 2 November 2014).

[5] W.F. Bond, R.L. Lammers, L.L. Spillane, R. Smith-Coggins, R. Fernandez and M.A. Reznek, "The use of simulation in emergency medicine: A research agenda", Acad Emerg Med, Society for Academic Emergency Medicine Simulation Task, F, 2007, pp. 353-363.

[6] H.T. Soltanian, "Surgical Simulation Update for Resident Education in Plastic Surgery", PRS Global Open, Publisher: Wolters Kluwer Health, Inc. on behalf of The American Society of Plastic Surgeons. Plast Reconstr Surg Glob Open 2016;4e773. www.PRSGobalOpen.com. Doi:10.1097/GOX. 0000000000000781. Published on line.

[7] K.R. Rosen, "The history of medical simulation", J Crit Care, 23(2), 2008, pp. 157-166.

[8] R.J. Scalese, Energising medical education through simulation: Powering minds, not just machines, 2009, 6th Asia Pacific Medical Education Conference Proceedings.

[9] A. Ziv, D. Erez, Y. Munz, A. Vardi, D. Barsuk, I. Levine and H. Berkenstadt. "The Israel Center for Medical Simulation: A paradigm for cultural change in medical education". Acad Med, 81(12), 2006, pp. 1091-1097.

[10] M.K. Mittal, K.R. Dumon, P.K. Edelson, N.M. Acero, D. Hashimoto, E. Danzer,and N.N. Williams, "Successful implementation of the American College of Surgeons Association of Program Directors in Surgery Surgical Skills Curriculum via a 4-week consecutive simulation rotation", Simulation Health, 7(3), 2012, pp. 147-154.

[11] P. Arbogast, and J. Rosen, Simulation in Plastic Surgery Training: Past, Present and Future, INTECH Open Access Publisher, 2012.

[12] C. Stern, A. Oliker, Z. Napier, J. Qualter, N. Deluccia, F. Sculli and A. Hazen, "Integration of surgical simulation in plastic surgery residency training", Stud Health Technol Inform, 173, 2012, pp. 497-499. http://www.ncbi.nlm.nih.gov/pubmed/ 22357043. (Accessed date: 14 April 2015). 
[13] ASERNIP-S (Australian Safety \& Efficacy Register of New Interventional Procedures - Surgical. The Royal Australasian College of Surgeons, 2007, Surgical simulation for training: Skills transfer to the operating room. http://www.surgeons.org/media/ 300327/Surgicalsimulation_sysematicreview.pdf. (Accessed date: 15 April 2015).

[14] R.M. Satava, "Emerging trends that herald the future of surgical simulation", Surg Clin North Am, 90(3), 2010, pp. 623-633.

[15] D.A. Cook, G. Bordage and H.G. Schmidt. "Description, justification and clarification: A framework for classifying the purposes of research in medical education" 2008, Medical Education 42, pp. 128-133.

[16] S.B. Issenberg, W.C. McGaghie, E.R. Petrusa, D. Lee Gordon and R.J. Scalese. "Features and uses of high-fidelity medical simulations that lead to effective learning: A BEME guide 4", 2004, pp. 1-37.

[17] C.P.G. Nel, M.J. Labuschagne and G.J. van Zyl. "Simulation in Plastic Surgery: A Research Agenda to Improve Teaching, Learning and Clinical Expertise/Professional Competence", 2016, Conference Proceedings, April 2016 IICE Conference, Ireland.

[18] J.M. Rosen, S.A. Long, D.M. McGrath and S.E. Greer, "Simulation in plastic surgery training and education: The path forward", Plast Reconstr Surg, 123(2), 2009, pp. 729-738; 739-740.

[19] ABMS \& ACGME (American Board of Medical Specialists and Accreditation Council for Graduate Medical Education) 2013, The Plastic Surgery Milestone Project: A Joint Initiative. http://search.acgme.org/search?q=cache:fAU8YXq

-D6kJ:www.acgme.org?acgmeweb. (Accessed date: 7 October 2014)

[20] M.W. Neumeister, "Technology and Education: The Future of Plastic Surgery Training”, PRS Global Open, Publisher: Wolters Kluwer Health, Inc. on behalf of The American Society of Plastic Surgeons. Plast Reconstr Surg Glob Open 2016;4e777. www.PRSGobalOpen.com. Doi:10.1097/GOX. 0000000000000780. Published online. 